\title{
The subjective size of visual stimuli affects the perceived duration of their presentation
}

\author{
FUMINORI ONO \\ Juntendo University, Tokyo, Japan \\ AND \\ JUN-ICHIRO KAWAHARA \\ National Institute of Advanced Industrial Science and Technology, Tsukuba, Japan
}

\begin{abstract}
The perception of time spent looking at a stimulus is lengthened or shortened when its physical attributes, such as area, differ from those of a comparison stimulus. We measured the perceived presentation duration of a visual object whose apparent area was altered by the Ebbinghaus illusion while its physical size remained invariant, so that a central circle surrounded by larger inducers appeared smaller than a same-size central circle surrounded by smaller inducers. The results showed that the perceived duration of presentation for apparently larger circles was longer than that of apparently smaller circles, although the actual area remained invariant across all circles. We concluded that the time perception process receives input from later visual processing.
\end{abstract}

The mechanism of time perception differs from that of visual or auditory perception because we do not have specific receptors that respond directly to time. According to Fraisse (1984), our experience of time depends on nontemporal events in a given interval. There is much evidence to show that temporal perception can be lengthened or shortened by nontemporal factors. For example, the perceived duration of briefly presented stimuli increases as their number (Mo, 1975; Mo \& Michalski, 1972), size (Long \& Beaton, 1980; Thomas \& Cantor, 1975), or complexity (Schiffman \& Bobko, 1974) increases. That is, the perceived duration of presentation increases when observers perceive the stimuli as being more numerous, larger, and/or more complex.

Although previous studies have suggested that time perception is influenced by visual processing, the critical stage of processing is not known. Visual processing can be broken into a series of steps; the corresponding visual cortices range in their operation from early to later stages. The visual areas operating at an early stage serve to extract local features and group them into symbolic tokens, and the higher-functioning areas operating at a later stage are responsible for shape inference and invariant object recognition (Marr, 1982). The present study sought to determine the part of the visual processing system that influences time perception.

To examine this topic, we combined the visual area size effect on time perception (Long \& Beaton, 1980; Thomas \& Cantor, 1975) and the Ebbinghaus illusion figure. In a typical procedure, from Thomas and Cantor's study of the visual area size effect on time perception, participants saw circles that varied in area and in presentation duration and reported both the area and the duration. The results showed that perceived presentation duration was directly related to the area of the stimulus: The perceived presentation duration increased as the circle increased in area. We measured the perceived duration of presentation of visual objects whose perceived area was altered by the Ebbinghaus illusion, wherein a central circle surrounded by large inducers appeared to be smaller than a same-size circle surrounded by small inducers. Previous studies have suggested that the Ebbinghaus illusion is to the result of active cognitive comparative processes rather than of peripheral physiological factors. For example, Coren and Enns (1993) manipulated the conceptual similarity of the inducers in the Ebbinghaus figure to the central object and found that this manipulation had an effect on the magnitude of the Ebbinghaus illusion. Specifically, in one experiment, they presented a line drawing of a dog as the central object and line drawings of shoes or horses as the surrounding inducers; the central dog was conceptually less similar to the surrounding shoes than to the surrounding horses, because both dogs and horses belong to the animal category. Results showed that the magnitude of the Ebbinghaus illusion was directly proportional to the rated conceptual similarity of the surrounding inducers to the central object. This finding clearly indicates the involvement of a higher level of visual processing in the Ebbinghaus illusion. In terms of the contribution of peripheral physiological factors, Coren, Ward, Porac, and Fraser (1978) examined the effect of optical blur on various types of visual illusions and found that increases in

F. Ono, fuminori@med.juntendo.ac.jp 


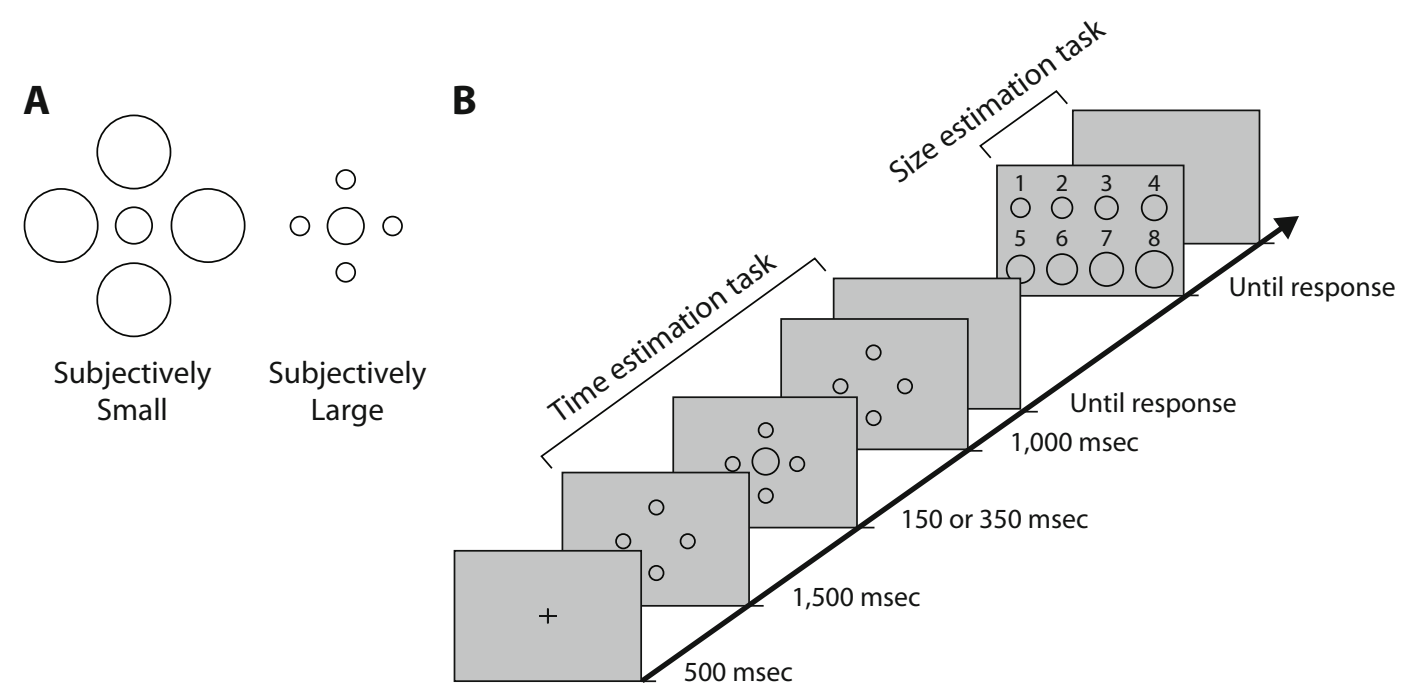

Figure 1. Examples of the stimuli. (A) The sequence of events in an experimental trial. (B) The participants categorized the duration of presentation of the central circle (time estimation task) and then the area of the central circle (size estimation task).

optical blur affected some types of geometric illusions, such as the Müller-Lyer illusion, but did not affect the strength of the Ebbinghaus illusion. On the basis of these findings, Coren and Enns suggested that the Ebbinghaus illusion is a prototype for size contrast illusions that affect cognitive judgment by introducing bias into the processing of information at a higher level of visual processing.

Thus, the present experiment, in which the effect of visual area on time perception was combined with Ebbinghaus figures, enabled us to examine whether time perception is affected by the processing of information at a later level of visual processing. If the time perception process shares the representation in which the size contrast illusion occurs, the perceived presentation duration of the central critical circle of the Ebbinghaus figure should remain the same when the subjective size of the central circle is increased or decreased by changing the size of the inducers. If, however, the time perception process mainly relies on the representation after the size contrast illusion has been established, the perceived presentation duration should be influenced by subjective size.

\section{EXPERIMENT 1}

\section{Method}

Participants. A total of 20 experimentally naive students, 19-23 years old, from Hiroshima University volunteered in return for course credit or payment. All had normal or corrected-tonormal visual acuity and had given their informed consent prior to participation.

Stimuli. Examples of the stimuli are shown in Figure 1A. The stimuli were composed of unfilled circles outlined in black on the gray background $\left(12.60 \mathrm{~cd} / \mathrm{m}^{2}\right)$ of a CRT monitor, with a viewing distance of approximately $60 \mathrm{~cm}$. There were two conditions: subjectively small and subjectively large circles. Four surrounding inducer circles were presented on the left, right, top, and bottom of the central circle in each condition. The diameter of the inducer circles was $4^{\circ}$ for the subjectively small condition and $1^{\circ}$ for the subjectively large condition. Under each condition, the diameter of the central circle was $2^{\circ}$ and the edge-to-edge distance of the surrounding circles from the central circle was $1^{\circ}$. The only difference between the two conditions was the area of the inducer circles; the area and presentation duration of the central circles were identical under both conditions.

Procedure. The experiment had both training and test phases. In the training phase, participants were taught to distinguish the presentation duration of a visual stimulus. Each participant initiated a training trial by pressing the space bar. Following a central fixation cross of 2,000-msec duration, only the central circle appeared for one of four random durations: $100,200,300$, or $400 \mathrm{msec}$. Participants were required to categorize presentation duration by pressing one of four keys (" 1 " for short and "4" for long). During the training phase, participants were instructed to establish their own subjective ratings for the duration of presentation of the central circle. No feedback was given in any part of the experiment.

After 60 training trials, participants received 120 test trials. The sequence of events for the test trials is shown in Figure 1B. The participants initiated a test trial by pressing the space bar. Following a central fixation cross of 500-msec duration, one of two sets of surrounding circles (those for the subjectively large or the subjectively small condition) was randomly displayed for $1,500 \mathrm{msec}$. The central circle appeared for either 150 or $350 \mathrm{msec}$. After the disappearance of the central circle, the inducer circles remained for $1,000 \mathrm{msec}$ and were then replaced by a blank screen. The participants categorized the duration of the central circle by pressing one of four keys. After the temporal categorization, eight variously sized comparison circles appeared. The diameters of the comparison circles varied in $0.1^{\circ}$ steps, with the diameter of the smallest circle $1.65^{\circ}$ and that of the largest $2.35^{\circ}$. From these eight alternatives, the participants chose a circle whose size seemed identical to that of the central circle by pressing one of eight keys ("1"-" 8 ").

\section{Results and Discussion}

The means for each condition in the time estimation task are presented in Figure 2A. An ANOVA with subjective size (subjectively small or large) and duration (150 or $350 \mathrm{msec}$ ) as within-subjects variables revealed significant main effects of subjective size $[F(1,19)=9.54$, $p<.01]$ and duration $[F(1,19)=377.14, p<.001]$. The interaction between subjective size and duration was not 

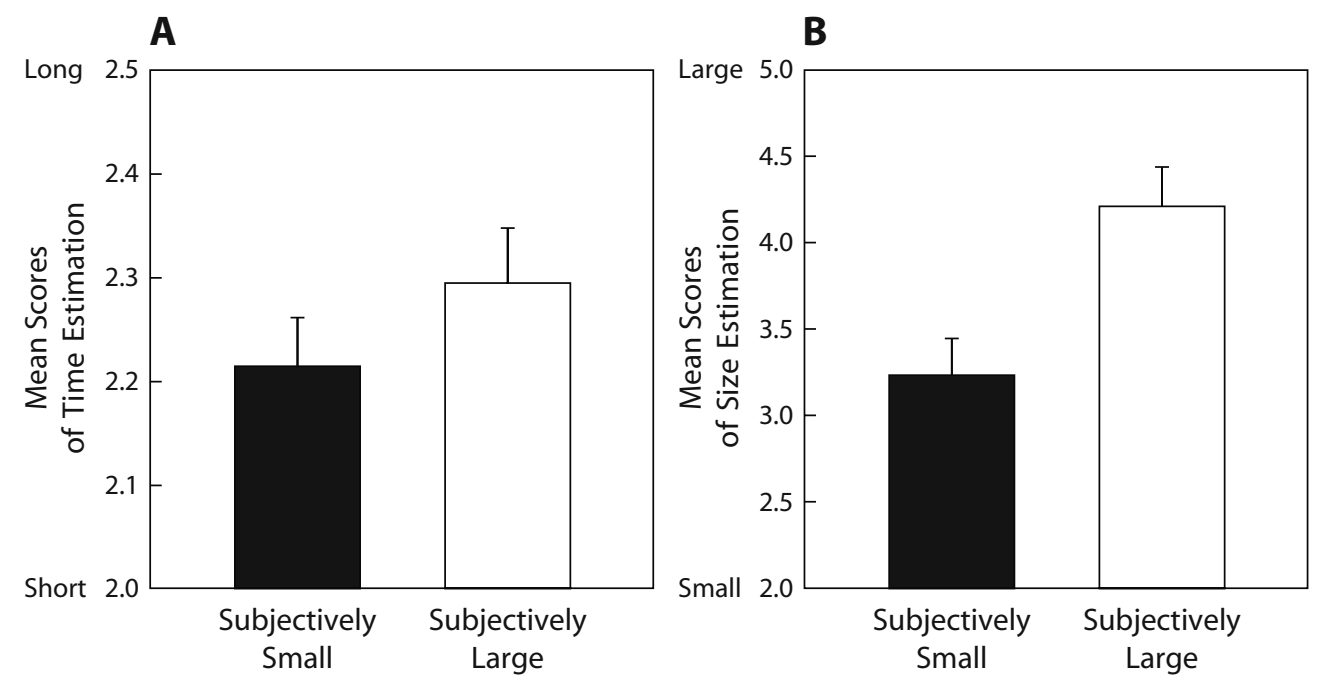

Figure 2. Results from Experiment 1. (A) The means for each condition in the time estimation task. (B) The means for each condition in the size estimation task. The error bars indicate standard errors.

significant $[F(1,19)=4.01, p=.06]$. The main effect of subjective size indicated that time perception was influenced by apparent area. Specifically, the perceived presentation duration of apparently large stimuli was longer than that of apparently small stimuli, although the actual area remained invariant. The mean scores of the time and $j$ size estimation tasks for each duration under each subjective size condition are presented in Table 1.

The means for each condition in the size estimation task are presented in Figure 2B. An ANOVA with subjective size and duration as within-subjects variables once again revealed significant main effects of subjective size $[F(1,19)=46.92, p<.001]$ and duration $[F(1,19)=$ $19.82, p<.001]$. The interaction between them was not significant $[F(1,19)=1.80, p=.19]$. The main effect of duration is consistent with the results of a previous study reporting that perceived area increased when participants perceived the presented duration of the stimuli as longer (Thomas \& Cantor, 1975). The main effect of subjective size suggests that the central circle was perceived as larger under the subjectively large condition than under the subjectively small condition. This confirmed that the apparent area of the central circle changed because of the Ebbinghaus illusion.

\section{EXPERIMENT 2}

In Experiment 1, the time estimation and size estimation tasks were presented consecutively, so participants could have estimated the central area during the time estimation task. If this were the case, variations in perceived duration might have been influenced by the size estimation, so that they were not pure reflections of time perception. Therefore, in a second experiment we ran the time estimation and size estimation tasks in separate blocks. If variations of perceived duration were due to the misattribution of size estimation rather than of time perception, the perceived duration under the subjectively large condition would be the same as that under the subjectively small condition. However, if apparent area influenced time perception even when participants were not engaged in the size estimation task, the pattern of results would be consistent with that of Experiment 1, and the perceived duration under the subjectively large condition would be longer than that under the subjectively small condition.

\section{Method}

Participants. A total of 20 experimentally naive students, 19-24 years old, from Hiroshima University volunteered in return for course credit or payment. All had normal or corrected-tonormal visual acuity and had given their informed consent prior to participation.

Stimuli and Procedure. All aspects of this experiment were the same as in Experiment 1, except that the time estimation and size estimation tasks were conducted in separate blocks. After 60 training trials, the participants performed the time estimation block (120 trials). After its completion, they were given instructions for the size estimation task. In particular, they were told to not estimate the area of the circle until they were given this instruction. In the size estimation block ( 40 trials), the same sequence of events as in the time estimation block was presented again, but this time the participants performed the size estimation task.

\section{Results and Discussion}

The means for each condition in the time estimation task are presented in Figure 3A. An ANOVA with subjec-

Table 1

Mean Scores of the Time and Size Estimation Tasks for Each Duration Under Each Subjective Size Condition in Experiment 1

\begin{tabular}{|c|c|c|c|c|c|c|c|c|}
\hline \multirow[b]{3}{*}{ Duration } & \multicolumn{4}{|c|}{ Time Estimation } & \multicolumn{4}{|c|}{ Size Estimation } \\
\hline & \multicolumn{2}{|c|}{$\begin{array}{c}\text { Subjectively } \\
\text { Small }\end{array}$} & \multicolumn{2}{|c|}{$\begin{array}{c}\text { Subjectively } \\
\text { Large }\end{array}$} & \multicolumn{2}{|c|}{$\begin{array}{c}\text { Subjectively } \\
\text { Small }\end{array}$} & \multicolumn{2}{|c|}{$\begin{array}{c}\text { Subjectively } \\
\text { Large }\end{array}$} \\
\hline & $M$ & $S E$ & $M$ & $\overline{S E}$ & $M$ & $S E$ & $M$ & $S E$ \\
\hline 1 & 1.47 & 0.0 & 1.4 & 0. & 3.12 & 0.22 & 4.04 & 0.22 \\
\hline $350 \mathrm{msec}$ & 2.96 & 0.07 & 3.09 & 0.06 & 3.33 & 0.21 & 4.37 & 0.23 \\
\hline
\end{tabular}



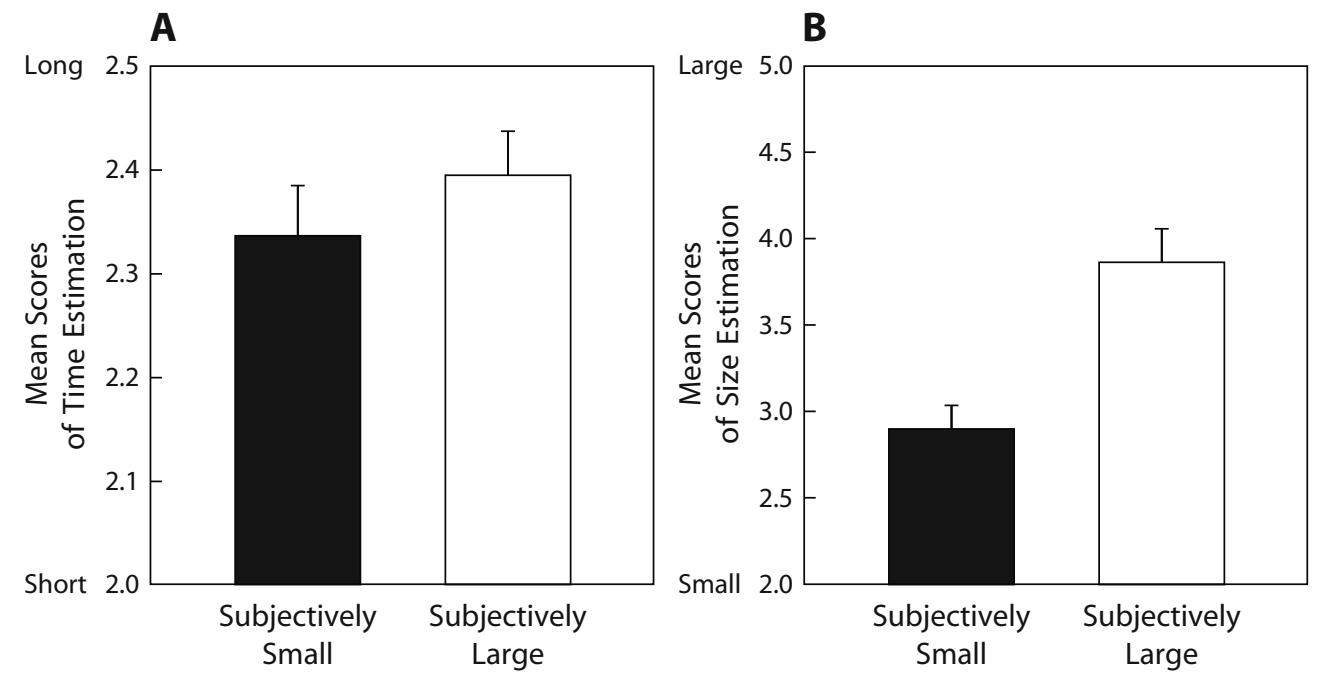

Figure 3. Results from Experiment 2. (A) The means for each condition in the time estimation task. (B) The means for each condition in the size estimation task. The error bars indicate standard errors.

tive size (subjectively small or large) and duration (150 or $350 \mathrm{msec}$ ) as within-subjects variables revealed significant main effects of subjective size $[F(1,19)=5.97$, $p<.05]$ and duration $[F(1,19)=504.82, p<.001]$. The interaction between subjective size and duration was significant $[F(1,19)=5.99, p<.05]$. This interaction suggests that the effect of subjective size is larger when there is a longer duration. In addition, the main effect of subjective size suggests that apparent area influences time perception even when participants are not engaged in the size estimation task. The mean scores of the time and size estimation tasks for each duration under each subjective size condition are presented in Table 2 .

Note that the mean perceived durations in Experiment 2 were longer than the perceived durations of the same stimuli in Experiment 1. A possible explanation for this increase is that perceived duration is influenced by sharing attentional resources between temporal and nontemporal processing (i.e., the attentional allocation model; see, e.g., Brown, 1985). According to this view, greater attention devoted to temporal processing will result in a longer perceived duration. In Experiment 2, there was no concurrent task (size estimation); therefore, attentional resources should have been fully devoted to temporal processing during the time estimation task, resulting in an increase in perceived duration.

The means for each condition in the size estimation task are presented in Figure 3B. An ANOVA with subjective size and duration as within-subjects variables revealed a significant main effect of subjective size $[F(1,19)=35.80, p<$ $.001]$, but the main effect of duration was not significant $[F(1,19)=1.06, p=.31]$, nor was the interaction between those factors $[F(1,19)=0.32, p=.57]$. The analysis of subjective size suggests that the apparent area of the central circle changed as a result of the Ebbinghaus illusion.

The results of Experiment 2 serve two purposes. First, the novel finding from Experiment 1 is clearly replicated, confirming that perceived duration was longer for appar- ently larger stimuli than for apparently smaller stimuli. Second, the effect was reproduced even when participants were not engaged in the size estimation task within the same block, eliminating the possibility that the present finding was influenced by knowing that a size estimation task was to follow.

\section{EXPERIMENT 3}

In Experiments 1 and 2, we found that perceived duration was influenced by subjective size. An alternative explanation for this effect is an attentional allocation effect from the surrounding inducers. Specifically, when surrounding inducers are large, they should compete for attentional allocation with the central critical circle, whereas with small inducers, there should be less competition. With less competition, more attentional resources can be allocated to the critical circle, resulting in a longer perceived duration. This alternative is viable, on the grounds that some research has shown that the perceived duration of an attended stimulus is longer than that of an unattended stimulus (e.g., Enns, Brehaut, \& Shore, 1999; Ono, Yamada, Chujo, \& Kawahara, in press). If this were the case, variations of perceived duration might have been influenced by attentional allocation. Such a possibility would detract from our claim that subjective size affects

Table 2

Mean Scores of the Time and Size Estimation Tasks for Each Duration Under Each Subjective Size Condition in Experiment 2

\begin{tabular}{|c|c|c|c|c|c|c|c|c|}
\hline \multirow[b]{3}{*}{ Duration } & \multicolumn{4}{|c|}{ Time Estimation } & \multicolumn{4}{|c|}{ Size Estimation } \\
\hline & \multicolumn{2}{|c|}{$\begin{array}{l}\text { Subjectively } \\
\text { Small }\end{array}$} & \multicolumn{2}{|c|}{$\begin{array}{l}\text { Subjectively } \\
\text { Large }\end{array}$} & \multicolumn{2}{|c|}{$\begin{array}{l}\text { Subjectively } \\
\text { Small }\end{array}$} & \multicolumn{2}{|c|}{$\begin{array}{c}\text { Subjectively } \\
\text { Large }\end{array}$} \\
\hline & $M$ & $\overline{S E}$ & $M$ & $S E$ & $M$ & $\overline{S E}$ & $M$ & $S E$ \\
\hline & 1.53 & 0. & 1.5 & 0 . & 2.8 & 0 . & 3.80 & 0.20 \\
\hline $350 \mathrm{msec}$ & 3.13 & 0.06 & 3.25 & 0.06 & 2.90 & 0.18 & 3.91 & 0.18 \\
\hline
\end{tabular}



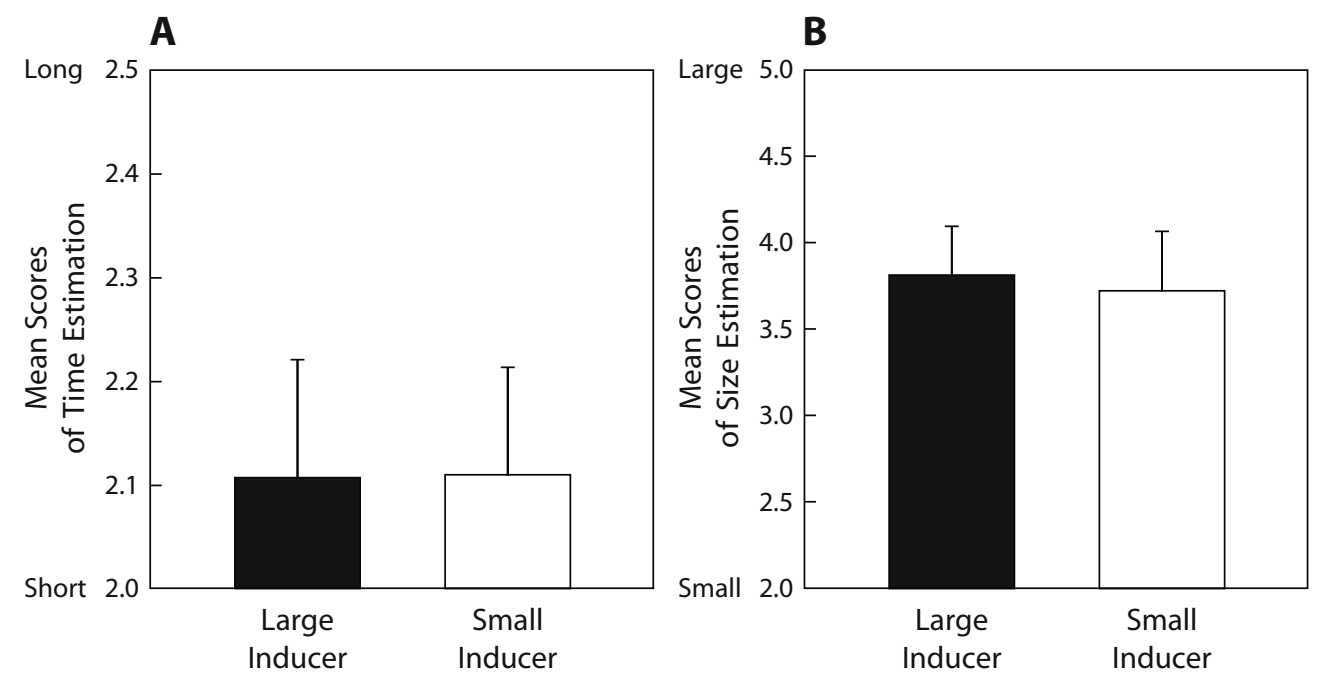

Figure 4. Results from Experiment 3. (A) The means for each condition in the time estimation task. (B) The means for each condition in the size estimation task. The error bars indicate standard errors.

perceived duration. To examine this attentional allocation alternative, we conducted Experiment 3, in which we manipulated the physical size of the central critical circles so that the perceived size of the circles was the same between the subjectively large (i.e., small inducers) and the subjectively small (i.e., large inducers) conditions. If variations of perceived duration were due to the attentional allocation from the surrounding inducers, perceived duration would be longer under the small-inducer condition than under the large-inducer condition. If, however, apparent area indeed influences time perception, the perceived duration under the small-inducer condition should be the same as under the large-inducer condition.

\section{Method}

Participants. A total of 12 experimentally naive students, 18-25 years old, from Hiroshima University volunteered in return for course credit or payment. All had normal or corrected-tonormal visual acuity and had given their informed consent prior to participation.

Stimuli and Procedure. All aspects of this experiment were the same as in Experiment 1, except that the size of the central circle was changed. The diameter of the central circle under the subjectively large condition was about $1.95^{\circ}$, and that under the subjectively small condition was about $2.05^{\circ}$. These diameters were chosen on the basis of the results of a pilot study in which the diameters of the central circles were adjusted so that the sizes of central circles under the small- and large-inducer conditions were subjectively equal.

\section{Results and Discussion}

The means for each condition in the time estimation task are presented in Figure 4A. An ANOVA with inducer size (small or large) and duration (150 or $350 \mathrm{msec}$ ) as within-subjects variables revealed a significant main effect of duration $[F(1,11)=133.20, p<.001]$. The main effect of inducer size was not significant $[F(1,11)=0.04$, $p=.83]$, nor was the interaction $[F(1,11)=1.59, p=$ .23]. The analysis of inducer size suggests that variations of perceived duration were not due to attentional allocation in this study. The mean scores of the time and size estimation tasks for each duration under each inducer size condition are presented in Table 3.

The means for each condition in the size estimation task are presented in Figure 4B. An ANOVA with inducer size and duration as within-subjects variables was conducted. This analysis revealed no significant results [inducer size, $F(1,11)=0.01, p=.91$; duration, $F(1,11)=0.22, p=$ .64 ; interaction, $F(1,11)=0.69, p=.42]$.

Experiment 3 showed that the perceived duration of a physically large stimulus did not differ from that of a physically small stimulus when the apparent areas of these stimuli were equated. This result eliminated the possibility that our findings for Experiments 1 and 2 were due to attentional allocation. Rather, we suggest that perceived duration was influenced by subjective size.

\section{GENERAL DISCUSSION}

The present study investigated the effect of subjective size on time perception. We manipulated the apparent area of visual stimuli by using a size contrast illusion (the Ebbinghaus illusion). The results revealed that time perception was influenced by subjective size: The perceived duration of apparently large stimuli was longer than that of apparently small stimuli, although the actual size of the stimuli remained invariant. This effect was obtained both

Table 3

Mean Scores of the Time and Size Estimation Tasks for Each Duration Under Each Inducer Size Condition in Experiment 3

\begin{tabular}{|c|c|c|c|c|c|c|c|c|}
\hline \multirow[b]{3}{*}{ Duration } & \multicolumn{4}{|c|}{ Time Estimation } & \multicolumn{4}{|c|}{ Size Estimation } \\
\hline & \multicolumn{2}{|c|}{$\begin{array}{l}\text { Large } \\
\text { Inducer }\end{array}$} & \multicolumn{2}{|c|}{$\begin{array}{l}\text { Small } \\
\text { Inducer }\end{array}$} & \multicolumn{2}{|c|}{$\begin{array}{l}\text { Large } \\
\text { Inducer }\end{array}$} & \multicolumn{2}{|c|}{$\begin{array}{c}\text { Small } \\
\text { Inducer }\end{array}$} \\
\hline & $M$ & $S E$ & $M$ & $S E$ & $M$ & $S E$ & $M$ & $S E$ \\
\hline $150 \mathrm{~m}$ & 1.44 & 0.11 & 1.41 & 0.10 & 3.73 & 0.30 & 3.54 & 0.32 \\
\hline $350 \mathrm{msec}$ & 2.77 & 0.14 & 2.80 & 0.13 & 3.86 & 0.29 & 3.89 & 0.35 \\
\hline
\end{tabular}


when time and size estimations were required simultaneously and when only the time estimation was required.

The present results clearly indicate that the perceived area of the central circle, rather than the area of the stimulus overall, affected the perception of presentation duration. If the present results were attributable to the area of the overall stimulus, including the surrounding inducers, the perceived duration in the subjectively small condition would have been longer than that in the subjectively large condition, because the area of the overall stimulus was larger in the former condition (Long \& Beaton, 1980; Thomas \& Cantor, 1975).

Importantly, the present findings demonstrate that time perception is influenced by a size contrast illusion that operates at higher levels of the visual system. Previous studies have divided the mechanisms of visual-geometric illusions into two classes (e.g., Coren et al., 1978): those created by optical and neural mechanisms, which can lead to illusory distortions, and those from cognitive judgmental effects, which introduce bias into the processing of information at higher levels. The size contrast illusion is the product of active cognitive comparative processes rather than peripheral physiological factors (Coren \& Enns, 1993; Jordan \& English, 1989), so our findings suggest that the time perception of visual events is influenced by higher-level systems in visual processing.

A related study examined how time perception is affected by lower-level systems of visual processing and used the kappa effect and the Müller-Lyer illusion (Lebensfeld $\&$ Wapner, 1968). The kappa effect is the phenomenon that occurs when two lights are presented successively in two different locations in space, in which an increase in the physical distance between the lights causes an increase in their apparent presentation duration. Lebensfeld and Wapner demonstrated that the kappa effect depends on the apparent distance induced by the Müller-Lyer illusion rather than on physical distance. This finding suggests that time perception is affected by lower-level visual processing, because it has been shown that a lower locus of visual processing is involved in the Müller-Lyer illusion (see, e.g., Coren et al., 1978).

In summary, the present results are the first to show the effect of a size contrast illusion (the Ebbinghaus illusion) on processing the temporal characteristics of a stimulus, indicating that the time perception process receives input not only from early visual processing but also from later processing. However, it should be noted that the present results do not imply that time perception takes place only at higher levels of visual processing. Rather, as has been suggested in recent studies, visual perception is achieved by a two-way interaction: Visual perception is not only built upon sensory evidence from lower-level processing, but is also guided by a top-down process that begins at higher-level cortices of the brain (see, e.g., Di Lollo, Enns, \& Rensink, 2000; Hochstein \& Ahissar, 2002; Lamme \& Roelfsema, 2000; Tong, 2003). Therefore, it is possible that time perception relies on both lower and higher levels of information processing. Indeed, recent neurophysiological studies have suggested that time per- ception depends on the integration of multiple neural systems (e.g., Meck, 2005). A definitive resolution regarding exactly how higher visual processes affect human temporal perception must await further research.

\section{AUTHOR NOTE}

This research was supported by a research fellowship to the first author from the Japan Society for the Promotion of Science for Young Scientists. We thank Jiro Gyoba and Rolf Ulrich for helpful comments on an earlier version of this article. Correspondence should be addressed to F. Ono, Department of Neurophysiology, School of Medicine, Juntendo University, 2-1-1 Hongo, Bunkyo-ku, Tokyo 113-8421, Japan (e-mail: fuminori@med.juntendo.ac.jp).

\section{REFERENCES}

Brown, S. W. (1985). Time perception and attention: The effects of prospective versus retrospective paradigms and task demands on perceived duration. Perception \& Psychophysics, 38, 115-124.

Coren, S., \& EnNs, J. T. (1993). Size contrast as a function of conceptual similarity between test and inducers. Perception \& Psychophysics, $\mathbf{5 4}, 579-588$

Coren, S., Ward, L. M., Porac, C., \& Fraser, R. (1978). The effect of optical blur on visual-geometric illusions. Bulletin of the Psychonomic Society, 11, 390-392.

Di Lollo, V., EnNs, J. T., \& RensinK, R. A. (2000). Competition for consciousness among visual events: The psychophysics of reentrant visual processes. Journal of Experimental Psychology: General, 129, 481-507.

Enns, J. T., Brehaut, J. C., \& Shore, D. I. (1999). The duration of a brief event in the mind's eye. Journal of General Psychology, 126, 355-372.

FraISSE, P. (1984). Perception and estimation of time. Annual Review of Psychology, 35, 1-36.

Hochstein, S., \& Ahissar, M. (2002). View from the top: Hierarchies and reverse hierarchies in the visual system. Neuron, 36, 791-804.

Jordan, K., \& English, P. W. (1989). Simultaneous sampling and length contrast. Perception \& Psychophysics, 46, 546-554.

Lamme, V. A., \& RoelfSema, P. R. (2000). The distinct modes of vision offered by feedforward and recurrent processing. Trends in Neurosciences, 23, 571-579.

Lebensfeld, P., \& WaPNER, S. (1968). Configuration and space-time interdependence. American Journal of Psychology, 81, 106-110.

Long, G. M., \& Beaton, R. J. (1980). The contribution of visual persistence to the perceived duration of brief targets. Perception \& Psychophysics, 28, 422-430.

MARR, D. (1982). Vision: A computational investigation into human representation and processing of visual information. San Francisco: Freeman.

MEcK, W. H. (2005). Neuropsychology of timing and time perception. Brain \& Cognition, 58, 1-8.

Mo, S. S. (1975). Temporal reproduction of duration as a function of numerosity. Bulletin of the Psychonomic Society, 5, 165-167.

Mo, S. S., \& MichaLSKI, V. A. (1972). Judgment of temporal duration of area as a function of stimulus configuration. Psychonomic Science, 27, 97-98.

Ono, F., Yamada, K., Chujo, K., \& Kawahara, J.-I. (in press). Feature-based attention influences later temporal perception. Perception \& Psychophysics.

Schiffman, H. R., \& Boвко, D. J. (1974). Effects of stimulus complexity on the perception of brief temporal intervals. Journal of Experimental Psychology, 103, 156-159.

Thomas, E. A. C., \& Cantor, N. E. (1975). On the duality of simultaneous time and size perception. Perception \& Psychophysics, 18, $44-48$.

Tong, F. (2003). Primary visual cortex and visual awareness. Nature Reviews Neuroscience, 4, 219-229.

(Manuscript received June 20, 2006; revision accepted for publication January 17, 2007.) 